\title{
Pneumocystis jirovecii pneumonia in a patient with melanoma treated with infliximab and corticosteroids for ipilimumab-associated colitis
}

\author{
Finbar Slevin ${ }^{1}$, Clive Mulatero² ${ }^{2}$ and Maria Marples ${ }^{2}$ \\ ${ }^{1}$ Specialty Registrar in Clinical Oncology, St James's Institute of Oncology, Leeds, United Kingdom \\ ${ }^{2}$ Consultant in Medical Oncology, St James's Institute of Oncology, Leeds, United Kingdom
}

\begin{abstract}
Immunotherapy has been successfully used in the management of malignancies including metastatic melanoma but is associated with autoimmune complications that may require corticosteroids and other immunosuppressive therapies to manage.

We describe a case of a woman with metastatic melanoma treated with ipilimumab who developed severe Pneumocystis jirovecci pneumonia (PCP) following treatment for ipilimumab-associated colitis with corticosteroids and infliximab. We outline the approach to diagnosis and management of this opportunistic infection.

PCP is an important infection to consider in the differential diagnosis for patients who are immunosuppressed and have an acute respiratory presentation. Early diagnosis and treatment is important especially since mortality is high in patients with cancer. Bronchoalveolar lavage and polymerase chain reaction is a useful diagnostic tool. First line therapy for PCP is trimethoprim/sulfamethoxazole. Patients with melanoma treated with immunomodulating therapies are at risk of autoimmune-related adverse events that may require powerful immunosuppressive treatments with the threat of subsequent opportunistic infection. Consideration should be given to PCP prophylaxis in these patients.
\end{abstract}

\begin{abstract}
Abbreviations: BAL: bronchoalveolar lavage; CD4+: cluster of differentiation 4 positive; CT: computed tomography; CTLA-4: cytotoxic T lymphocyte associated antigen-4; HIV: human immunodeficiency virus; PCP: Pneumocystis jirovecci pneumonia; PCR: polymerase chain reaction; TMP/SMX: trimethoprim/sulfamethoxazole; TNF-alpha: tumour necrosis factor-alpha
\end{abstract}

\section{Introduction}

Recently immunotherapy has been successfully used to manage metastatic melanoma and other cancers and oncologists should be aware of the potential complications resulting from management of immunotherapy toxicities. Pneumocystis jirovecci pneumonia (PCP) following treatment for autoimmune complications related to ipilimumab has been previously reported but only in a small number of cases [1]. We therefore outline the approach to diagnosis and management of this unusual opportunistic infection.

\section{Case presentation}

A 52-year-old woman presented with breathlessness. She had been found to have BRAF mutant metastatic melanoma five years previously. She had been treated with vemurafenib for two years and had had a right pneumonectomy for a single site of progression at the right lung hilum. She had continued vemurafenib but had progressed six months later, and had received three cycles of ipilimumab. The third cycle had been followed by an episode of terminal ileitis and pancolitis that had been treated with high dose corticosteroids (methylprednisolone followed by a slowly reducing course of prednisolone) and two doses of infliximab $5 \mathrm{mg} / \mathrm{kg}$.
Two months later, she was admitted with a five day history of worsening breathlessness, that was initially on exertion but then at rest. There was an associated dry cough and low grade fever. She was still taking prednisolone $25 \mathrm{mg} /$ day and one $500 \mathrm{mg}$ calcium $/ 400$ international units colecalciferol tablet per day but no other regular medications.

Clinical assessment revealed normal air entry on the left side of the chest with signs of a right pneumonectomy. She was tachycardic at 101 beats per minute, respiratory rate was raised at 24 per minute, oxygen saturation was reduced at $96 \%$ on $2 \mathrm{~L} /$ minute of oxygen. Chest radiograph demonstrated the left lung to be clear. Blood tests showed a white cell count of $14 \times 10^{9} / \mathrm{L}$ (normal range $4-11 \times 10^{9} / \mathrm{L}$ ) with a neutrophilia and $\mathrm{C}$ reactive protein of $22 \mathrm{mg} / \mathrm{L}$ (normal range $<10 \mathrm{mg} / \mathrm{L}$ ). Computed tomography pulmonary angiography excluded pulmonary embolus but did show new left sided patchy and widespread ground glass changes with mediastinal lymphadenopathy (Figure 1).

Treatment for community acquired pneumonia with intravenous piperacillin/tazobactam and clarithromycin was given but she failed to improve. Screens for atypical bacterial, viral and fungal pneumonia screens were negative for legionella, mycoplasma, psittacosis, Q fever,

Correspondence to: Dr. Finbar Slevin, Specialty Registrar in Clinical Oncology, St James's Institute of Oncology, Leeds, United Kingdom, E-mail: finbarslevin@nhs.net

Key words: ipilimumab, infliximab, melanoma, Pneumocystis jirovecci pneumonia Received: August 28, 2016; Accepted: September 17, 2016; Published: September 20, 2016 


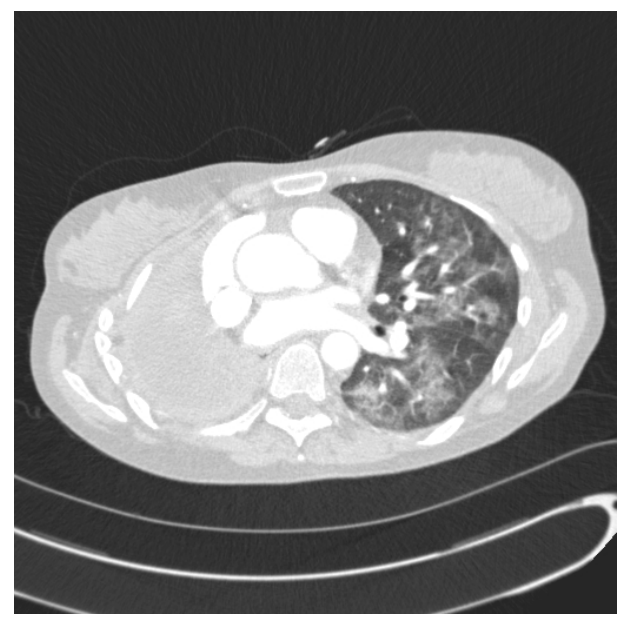

Figure 1. Axial computed tomography slice of the chest showing diffuse ground glass opacification within the left lung and right pneumonectomy.

Epstein-Barr virus, cytomegalovirus, respiratory viruses and aspergillus. Human immunodeficiency virus (HIV) screen was negative.

Bronchoscopy and bronchoalveolar lavage (BAL) was performed two days after admission and following this she deteriorated with rising fevers, increasing hypoxia and increasing tachycardia and tachypnoea.

Intravenous trimethoprim/sulfamethoxazole (TPM/SMX) was added to cover the possibility of PCP. Her dose of prednisolone was increased to $60 \mathrm{mg}$ per day. The BAL polymerase chain reaction (PCR) returned positive four days after the bronchoscopy for Pneumocystis jirovecci with a high organism load consistent with PCP.

The patient improved on TMP/SMX and was switched to an oral dose at day 7.

She was discharged at day 10 and completed a 14 day course of TMP/SMX in total. She had community monitoring of her full blood count, renal and liver biochemistry to check for toxicity associated with TMP/SMX. When reviewed in clinic two weeks later she had no clinical features to suggest relapse of PCP and had recovered her function back close to her baseline.

When seen at 4 months she was well with no clinical or radiological evidence of progression of her melanoma.

\section{Discussion}

Pneumocystis pneumonia (PCP) is caused by the unicellular fungus Pneumocystis jirovecii. Transmission of Pneumocystis cysts is by airborne inhalation and immunocompromised people are at risk of developing opportunistic PCP either by re-activation of latent organisms in the lung or by acute infection [2]. People with impaired $\mathrm{T}$ cell immunity are at highest risk, especially with CD4 positive $\mathrm{T}$ cell counts below $200 / \mu \mathrm{L}[3]$.

At risk groups for PCP include human immunodeficiency virus (HIV) infected individuals while non-HIV risk factors are immunodeficiency associated with cytotoxic or immunosuppressive therapies for solid and haematological malignancy, solid organ and bone marrow transplantation and inflammatory conditions [2]. Systemic corticosteroid use appears to predispose to PCP in solid tumours, especially for patients receiving high dose dexamethasone for central nervous system disease [4]. Patients treated with the anti-TNF drug infliximab for inflammatory bowel disease and rheumatological conditions have developed PCP. TNF-alpha is important in the immune response to infection, especially within the lung, but these patients are frequently also treated with corticosteroids [5,6]. Postmarketing surveillance has shown the incidence of PCP in patients treated with Infliximab to be $0.4 \%$ [7].

Ipilimumab is a monoclonal antibody that blocks cytotoxic $\mathrm{T}$ lymphocyte associated antigen-4 (CTLA-4) preventing inhibition of $\mathrm{T}$ cell anti-tumour response. As a consequence of this it is associated with autoimmune related adverse events including a spectrum of gastrointestinal toxicities. Colitis is seen in fewer than $10 \%$ of patients but can lead to bowel obstruction and perforation [8]. Management of grade 3 (increase in stool frequency of $>7$ per day, fever, severe abdominal pain or peritonism) and 4 (life-threatening) colitis involves excluding gastrointestinal infection and treating with high dose steroids for example intravenous methylprednisolone converting to a tapering dose of oral prednisolone over six to eight weeks with resolution. In the absence of improvement at 48 hours infliximab can be initiated. Ipilimumab should be permanently discontinued. Surgical input may be required in the presence of bowel obstruction or perforation [9].

Clinical presentation of PCP is non-specific with dry cough and progressive breathlessness typically over one to three weeks. Fever may be present. Acute breathlessness and pleuritic chest pain may herald development of pneumothorax. Examination findings include tachypnoea, tachycardia, hypoxia including exercise desaturation and cyanosis. Auscultation of the chest is generally unremarkable [10].

The typical chest radiograph feature is bilateral perihilar interstitial shadowing that becomes more diffuse over time but it may be normal especially early in the course of the disease. More unusual features are solitary or multiple nodules and pneumothoraces. Pleural effusions and lymphadenopathy are rare $[2,10]$. Computed tomography (CT) of the chest usually demonstrates extensive ground glass opacification but may also show cystic lesions and consolidation in non-HIV patients. In HIV patients ground glass opacification tends to be diffuse and inhomogeneous. In non-HIV patients this pattern is seen but there may be more sharply demarcated and lobular ground glass opacifications [11].

Light microscopy, immunofluorescent assays and polymerase chain reaction (PCR) are important diagnostic investigations in the context of non-specific clinical and radiological features. These can be performed on induced sputum, brochoalveolar lavage (BAL) or transbronchial lung biopsy samples. For microscopic examination staining is necessary since Pneumocystis cannot be cultured [10]. The least invasive method of investigation is testing of sputum samples induced using hypertonic saline. However microscopic diagnosis using induced sputum and BAL samples is less sensitive in non-HIV cases of Pneumocystis, where fewer organisms tend to be present and sensitivity may be less than $50 \%$ [12]. PCR of induced sputum or BAL has been shown to be more useful with sensitivity of $87 \%$, specificity of $92 \%$ and negative predictive value of over $98 \%$. Samples, especially non-invasive induced sputum, should be taken prior to or soon after treatment for PCP is started to reduce the risk of false negative results. One drawback of PCR is the detection of Pneumocystis asymptomatic colonization. However the high negative predictive value of PCR means that a negative result indicates treatment for PCP can be stopped [13]. The accuracy of PCR also means lung biopsy for diagnosis is rarely required.

The British HIV Association stratifies PCP infection by severity and this determines the recommended treatment. Severity depends on symptoms, degree of hypoxia and chest radiographic features. Mild 
disease would be suggested by breathlessness only on exertion, pulse oximetry $>96 \%$ on room air, $\mathrm{PaO}_{2}>11 \mathrm{kPa}$ on arterial blood gas and normal or minimal changes on chest radiograph. Moderate to severe disease is on a spectrum from breathlessness on minimal exertion to at rest. The presence of fever suggests more severe disease. In addition pulse oximetry $<96 \%$ or $\mathrm{PaO}_{2}<11 \mathrm{kPa}$ and diffuse chest radiographic

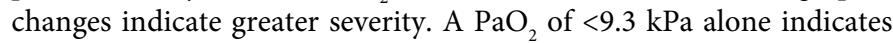
severe disease [14]. The patient in our case had severe PCP given she had breathlessness at rest, fever and hypoxia.

Pneumocystis is an atypical fungus and due to the presence of cholesterol in its cell membrane does not respond to azoles and amphotericin B. These drugs target ergosterol, a component of the cell membrane in fungi such as Candida and Aspergillus species [15]. First line recommended treatment for PCP is trimethoprim/ sulfamethoxazole (TMP/SMX) at $120 \mathrm{mg} / \mathrm{kg}$ per day intravenously in three to four divided doses in moderate to severe infection. Duration of therapy is recommended to be 14 to 21 days but patients demonstrating a clinical improvement may complete the course with an oral dose of TMP/SMX. Mild infection may be treated with a lower dose of $90 \mathrm{mg} / \mathrm{kg}$ orally. Alternative treatments for patients unable to take TMP/SMX or who fail to respond to initial therapy include clindamycin, primaquine, pentamidine, dapsone and atovaquone. The British HIV Association recommends that glucose-6-phosphate dehydrogenase deficiency should be checked for prior to treatment with TMP/SMX, dapsone or primaquine given the risk of haemolysis but treatment should not be delayed while awaiting the result. Patients with moderate to severe disease or those with $\mathrm{PaO}_{2}<9.3 \mathrm{kPa}$ should receive corticosteroids starting at prednisolone $40 \mathrm{mg}$ twice daily on days $1-5,40 \mathrm{mg}$ once daily on days 6-10 and $20 \mathrm{mg}$ once daily on days 11-21 [14].

There is evidence of transmission of Pneumocystis between people including strains resistant to TMP/SMX, a first line treatment for PCP. Therefore it may be sensible to limit contact of infected individuals with other immunocompromised patients during the acute phase of the illness [16].

Pneumocystis prophylaxis is used successfully for HIV patients with low CD4 counts and for organ transplant recipients. It has been suggested prophylaxis is considered for patients receiving the equivalent of $20 \mathrm{mg}$ prednisolone for more than one month and for inflammatory bowel disease patients treated with three immunosuppressive therapies including anti-TNF therapy. However there remains a lack of consensus guidelines relating to $\mathrm{PCP}$ prophylaxis for patients receiving immunosuppressive therapy outside of the organ transplantation setting. The reduction in risk of PCP also has to be balanced against the risk of toxicity from the commonly used drugs for prophylaxis. For example TMP/SMX can cause hypersensitivity, severe skin rashes that may progress to Stevens-Johnson syndrome or toxic epidermal necrolysis, leukopenia, renal and hepatic dysfunction. Dapsone may cause hypersensitivity as well as haemolysis and methaemoglobinaemia [17].

The mortality associated with PCP in non-HIV patients is greater and has been found to range from $30-60 \%$ compared to mortality in HIV positive patients of $10-20 \%$. The disease appears to have a more rapid onset and progression in non-HIV individuals and greater need for intensive care admission and mechanical ventilation. Patients with cancer have poorer outcomes than those who develop PCP while on immunosuppressive treatments for connective tissue disorders or organ transplantation $[4,11,18,19]$. Infection in non-HIV patients tends to be associated with fewer Pneumocystis organisms but larger numbers of neutrophils and greater pulmonary inflammation, which correlates with more severe hypoxaemia and poorer outcomes [20].

\section{Conclusion}

PCP is an important opportunistic infection to consider in the differential diagnosis for patients who are immunosuppressed and have an acute respiratory presentation. Early diagnosis and treatment is important especially since mortality is high in patients with cancer. Patients with melanoma treated with immunomodulating therapies are at risk of autoimmune-related adverse events that may require powerful immunosuppression to manage with the threat of subsequent opportunistic infection. Consideration should be given to PCP prophylaxis in these patients.

\section{Declarations}

Written informed consent was obtained from the patient described in this case report.

The authors declare that there is no conflict of interest regarding the publication of this paper.

The authors declare that no specific sources of funding were sought.

The authors declare that there are no financial or non-financial competing interests.

\section{References}

1. Arriola E, Wheater M, Krishnan R, Smart J, Foria V, et al. (2015) Immunosuppression for ipilimumab-related toxicity can cause pneumocystis pneumonia but spare antitumour control. Oncoimmunology 4: e1040218. [Crossref]

2. Sokulska M, Kicia M, Weso§̊,owska M, Hendrich AB (2015) Pneumocystis jirovecii-from a commensal to pathogen: clinical and diagnostic review. Parasitol Res 114: 3577-3585. [Crossref]

3. Phair J, Muñoz A, Detels R, Kaslow R, Rinaldo C, et al. (1990) The risk of Pneumocystis carinii pneumonia among men infected with human immunodeficiency virus type 1 . Multicenter AIDS Cohort Study Group. N Engl J Med 322: 161-165. [Crossref]

4. Sepkowitz KA (2002) Opportunistic Infections in Patients with and Patients without Acquired Immunodeficiency Syndrome. Clin Infect Dis 34: 1098-1107. [Crossref]

5. van Dartel SA, Fransen J, Kievit W, Flendrie M, den Broeder AA, et al. (2012) Difference in the risk of serious infections in patients with rheumatoid arthritis treated with adalimumab, infliximab and etanercept: results from the Dutch Rheumatoid Arthritis Monitoring (DREAM) registry. Ann Rheum Dis 72: 895-900. [Crossref]

6. Toussi SS, Pan N, Walters HM, Walsh TJ (2013) Infections in Children and Adolescents With Juvenile Idiopathic Arthritis and Inflammatory Bowel Disease Treated With Tumor Necrosis Factor-a Inhibitors: Systematic Review of the Literature. Clin Infect Dis 57: 1318-1330. [Crossref]

7. Takeuchi T, Tatsuki Y, Nogami Y, Ishiguro N, Tanaka Y, et al. (2008) Postmarketing surveillance of the safety profile of infliximab in 5000 Japanese patients with rheumatoid arthritis. Ann Rheum Dis 67: 189-194. [Crossref]

8. Ibrahim RA, Berman DM, DePril V, Humphrey RW, Chen T, et al. (2011) Ipilimumab safety profile: summary of findings from completed trials in advanced melanoma. In American Society of Clinical Oncology Annual Meeting. Chicago, IL, USA.

9. Klair JS, Girotra M, Hutchins LF, Caradine KD, Aduli F, et al. (2016) IpilimumabInduced Gastrointestinal Toxicities: A Management Algorithm. Dig Dis Soc 61: 2132 2139. [Crossref]

10. Thomas CF Jr, Limper AH (2004) Pneumocystis pneumonia. N Engl J Med 350: $2487-$ 2498. [Crossref]

11. Tasaka S, Tokuda H, Sakai F, Fujii T, Tateda K, et al. (2010) Comparison of Clinical and Radiological Features of Pneumocystis Pneumonia Between Malignancy Cases and Acquired Immunodeficiency Syndrome Cases: A Multicenter Study. Intern Med 49: 273-281. [Crossref]

12. Lipschik GY, Gill VJ, Lundgren JD, Andrawis VA, Nelson NA, et al. (1992) Improved diagnosis of Pneumocystis carinii infection by polymerase chain reaction on induced sputum and blood. Lancet 340: 203-206. [Crossref] 
13. Azoulay E, Bergeron A, Chevret S, Bele N, Schlemmer B, et al. (2009) Polymerase Chain Reaction for Diagnosing Pneumocystis Pneumonia in Non-HIV Immunocompromised Patients With Pulmonary Infiltrates. Chest 135: 655-661. [Crossref]

14. Nelson M, Dockrell D, Edwards S; BHIVA Guidelines Subcommittee, Angus B, et al. (2011) British HIV Association and British Infection Association guidelines for the treatment of opportunistic infection in HIV-seropositive individuals. HIV Med 12 Suppl 2:1-140. [Crossref]

15. Macreadie IG1, Johnson G, Schlosser T, Macreadie PI (2006) Growth inhibition of Candida species and Aspergillus fumigatus by statins. FEMS Microbiol Lett 262: 9-13. [Crossref]

16. Barry SM, Johnson MA (2001) Pneumocystis carinii pneumonia: a review of current issues in diagnosis and management. HIV Med 2: 123-132. [Crossref]

17. Green H, Paul M, Vidal L, Leibovici L (2014) Prophylaxis for Pneumocystis pneumonia (PCP) in non-HIV immunocompromised patients. Cochrane Database Syst Rev 3: CD005590. [Crossref]

18. Arozullah AM, Yarnold PR, Weinstein RA, Nwadiaro N, Mcllraith TB, et al. (2000) A New Preadmission Staging System for Predicting Inpatient Mortality from HIVassociated Pneumocystis carinii Pneumonia in the Early Highly Active Antiretroviral Therapy (HAART) Era. Am J Respir Crit Care Med 161: 1081-1086. [Crossref]

19. Roblot F1, Godet C, Le Moal G, Garo B, Faouzi Souala M, et al. (2002) Analysis of Underlying Diseases and Prognosis Factors Associated with Pneumocystis carinii Pneumonia in Immunocompromised HIV-Negative Patients. Eur J Clin Microbiol Infect Dis 21: 523-531. [Crossref]

20. Limper AH, Offord KP, Smith TF, Martin WJ $2^{\text {nd }}$ (1989) Pneumocystis carinii pneumonia. Differences in lung parasite number and inflammation in patients with and without AIDS. Am Rev Respir Dis 140: 1204-1209. [Crossref]

Copyright: $\mathbb{C} 2016$ Slevin F. This is an open-access article distributed under the terms of the Creative Commons Attribution License, which permits unrestricted use, distribution, and reproduction in any medium, provided the original author and source are credited. 